\title{
Affine permutations and rational slope parking functions
}

\author{
Eugene Gorsky ${ }^{1}$ \\ Mikhail Mazin ${ }^{2}$ \\ Monica Vazirani ${ }^{3}$ \\ ${ }^{1}$ Columbia University, New York, New York, USA \\ ${ }^{2}$ Kansas State University, Manhattan, Kansas, USA \\ ${ }^{3}$ University of California at Davis, Davis, California, USA
}

\begin{abstract}
We introduce a new approach to the enumeration of rational slope parking functions with respect to the area and a generalized dinv statistics, and relate the combinatorics of parking functions to that of affine permutations. We relate our construction to two previously known combinatorial constructions: Haglund's bijection $\zeta$ exchanging the pairs of statistics (area, dinv) and (bounce, area) on Dyck paths, and Pak-Stanley labeling of the regions of $k$-Shi hyperplane arrangements by $k$-parking functions. Essentially, our approach can be viewed as a generalization and a unification of these two constructions.

Résumé. Nous introduisons une nouvelle approche pour énumérer les fonctions parking pente rationelles, en se focalisant sur la statistique area ainsi qu'une généralisation de la statistique dinv. Nous rapprochons la combinatoire des fonctions de parking à celle des permutations affines. Nous ramenons la construction à deux constructions déjà connues: la bijection d'Haglund $\zeta$ entre les statistiques (area, dinv) et (bounce, area) sur les chemins de Dyck, et l'étiquetage de Pak-Stanley des arrangements des $k$-Shi hyperplans par les $k$-fonctions de parking. Essentiellement, notre approche généralise et unifie ces deux constructions.
\end{abstract}

Keywords: Parking functions, affine permutations

\section{Introduction}

Parking functions are prevalent in modern combinatorics. There is a natural action of the symmetric group on parking functions, and the orbits are labeled by the non-decreasing parking functions which correspond naturally to Dyck paths. This provides a link between parking functions and various combinatorial objects counted by Catalan numbers. In a series of papers Garsia, Haglund, Haiman, et al. [10, 11], related the combinatorics of Catalan numbers and parking functions to the space of diagonal harmonics. There are also deep connections to the geometry of the Hilbert scheme.

Since the works of Pak and Stanley [16], Athanasiadis and Linusson [4] , it became clear that parking functions are tightly related to the combinatorics of the affine symmetric group. In particular, they provided two different bijections between the parking functions and the regions of Shi hyperplane arrangement. It has been remarked in [2, 6, 15] that the inverses of the affine permutations labeling the minimal alcoves in Shi regions belong to a certain simplex $D_{n}^{n+1}$, which is isometric to the $(n+1)$-dilated 


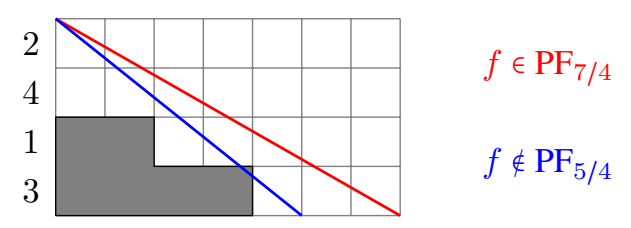

Fig. 1: The labeled diagram for the parking function $f=(2040)$.

fundamental alcove. As a result, the alcoves in $D_{n}^{n+1}$ can be labeled by parking functions in two different ways.

Many of the concepts above have "rational" counterparts corresponding to a coprime pair $(m, n)$, for which the classical case is $(n+1, n)$. In [8] and [9] the first and the second authors proved that the rational Dyck paths label the affine cells in a certain algebraic variety, the compactified Jacobian of a plane curve singularity with one Puiseaux pair $(m, n)$. This work was generalized by Hikita in [12] who proved that $\mathrm{m} / \mathrm{n}$ parking functions naturally label the cells in a certain Springer fibre in the affine flag variety for the affine symmetric group $\widetilde{S}_{n}$. As a consequence of this paper, we can use our construction to prove new formulas for the Poincaré polynomials of certain affine Springer fibers relying on the work of [8] and [9].

The present paper is dedicated to the systematic study of the $m / n$ parking functions in terms of the affine symmetric group $\widetilde{S}_{n}$. We consider the set $\widetilde{S}_{n}^{m}$ of all affine permutations in $\widetilde{S}_{n}$ with no inversions of height $m$ and two maps $\mathcal{A}$ and $\mathcal{P} \mathcal{S}$ from $\widetilde{S}_{n}^{m}$ to the set of $m / n$ parking functions $\mathcal{P} \mathcal{F}_{m / n}$. When one composes $\mathcal{P S}$ with $\mathcal{A}^{-1}$, one recovers the zeta map $\zeta$ of Haglund, which was used to give a combinatorial proof of the weak symmetry property of the $q, t$-Catalan numbers.

\section{Acknowledgements}

The authors would like to thank the American Institute of Mathematics for their hospitality, and D. Armstrong, F. Bergeron, S. Fishel, I. Pak, R. Stanley, V. Reiner, B. Rhoades, A. Varchenko, G. Warrington and N. Williams for useful discussions and suggestions. E. G. was partially supported by the grants RFBR-1301-00755, NSh-4850.2012.1. M. V. was partially supported by the grant NSA MSP H98230-12-1-0232.

\section{Tools and definitions}

We start with a brief review of the definitions and basic results involving parking functions, affine permutations, and hyperplane arrangements, which will play the key role in our constructions.

\subsection{Parking Functions}

Definition 2.1 A function $f:\{1, \ldots, n\} \rightarrow \mathbb{Z}_{\geq 0}$ is called an $m / n$-parking function if the Young diagram with row lengths equal to $f(1), \ldots, f(n)$ put in decreasing order, bottom to top, fits under the diagonal in an $n \times m$ rectangle. The set of such functions is denoted by $\mathcal{P} \mathcal{F}_{m / n}$.

We will often use the notation $f=|f(1) f(2) \ldots f(n)\rangle$ for parking functions.

Example 2.2 Consider the function $f:\{1,2,3,4\} \rightarrow \mathbb{Z}_{\geq 0}$ given by $f(1)=2, f(2)=0, f(3)=4$, and $f(4)=0$ (i.e. $f=(2040)$ ). The corresponding Young diagram fits under the diagonal in a $4 \times 7$ rectangle, but does not fit under the diagonal in a $4 \times 5$ rectangle. Thus, $f \in P F_{7 / 4}$ but $f \notin P F_{5 / 4}$ (see Figure 7 ). 
Equivalently, a function $f:\{1, \ldots, n\} \rightarrow \mathbb{Z}_{\geq 0}$ belongs to $\mathcal{P} \mathcal{F}_{m / n}$ if and only if it satisfies

$$
\forall i \in\{0, \ldots, n-1\}, \sharp\left\{k \in\{1, \ldots, n\} \mid f(k) \leq \frac{i m}{n}\right\} \geq i+1 .
$$

Let $P: \mathcal{P} \mathcal{F}_{m / n} \rightarrow Y_{m, n}$ denote the natural map from the set of parking functions to the set $Y_{m, n}$ of Young diagrams that fit under diagonal in an $n \times m$ rectangle. To recover a parking function $f \in \mathcal{P} \mathcal{F}_{m / n}$ from the corresponding Young diagram $P(f)$ one needs some extra information. Lengths of the rows of $P(f)$ correspond to the values of $f$, but one needs also to assign the preimages to them. That is, one should label the rows of $P(f)$ by integers $1,2, \ldots, n$. Note that if $P(f)$ has two rows of the same length, then the order of the corresponding labels does not matter. One should choose one of the possible orders. We choose the decreasing order (read from bottom to top).

Definition 2.3 Let $\widehat{Y}_{m, n}$ denote the set of couples $(D, \tau)$ of a Young diagram $D \in Y_{m, n}$ and a (finite) permutation $\tau \in S_{n}$, such that if $k$ th and $(k+1)$ th rows of $D$ have the same length, then $\tau(k+1)<\tau(k)$. We will refer to $\tau$ as the row-labeling of $D$.

Note that $\tau \in S_{n}$ is the permutation of maximal length such that $f \circ \tau$ is non-increasing.

Example 2.4 In Example 2.2, one has $\tau=[3,1,4,2]$, so $f \circ \tau=(2040) \circ[3,1,4,2]=(4200)$.

We get the following lemma:

Lemma 2.5 The set of $m / n$-parking functions $\mathcal{P} \mathcal{F}_{m / n}$ is in bijection with the set of labeled Young diagrams $\widehat{Y}_{m, n}$.

Remark 2.6 Note that for $m=n+1$ the set $\mathcal{P} \mathcal{F}_{m / n}$ is exactly the set of classical parking functions $\mathcal{P} \mathcal{F}$, and for $m=k n+1$ it is the set of $k$-parking functions $\mathcal{P F}_{k}$ (e.g. [10]).

From now on we will assume that $m$ and $n$ are coprime, so there are no lattice points on the diagonal of $n \times m$ rectangle. By abuse of notation, we will call a non-decreasing parking function increasing. The number of increasing parking functions equals the generalized Catalan number $\sharp Y_{m, n}=\frac{1}{n+m}\left(\begin{array}{c}n+m \\ n\end{array}\right)$. The number of all parking functions $\sharp \mathcal{P} \mathcal{F}_{m / n}$ equals $m^{n-1}$. (See [4].)

\subsection{Affine Permutations}

Definition 2.7 The affine symmetric group $\widetilde{S}_{n}$ is generated by $s_{1}, \ldots, s_{n-1}, s_{0}$ subject to the relations

$$
\begin{array}{ll}
s_{i}^{2}=1, & \\
s_{i} s_{j}=s_{j} s_{i} & \text { for } i-j \not \equiv \pm 1 \bmod n, \\
s_{i} s_{j} s_{i}=s_{j} s_{i} s_{j} & \text { for } i-j \equiv \pm 1 \bmod n(\text { if } n>2) .
\end{array}
$$

Let

$$
\overline{\mathbf{x}}=\left(\begin{array}{c}
x_{1} \\
\vdots \\
x_{n}
\end{array}\right), \quad V:=\left\{\overline{\mathbf{x}} \in \mathbb{R}^{n} \mid x_{1}+\ldots+x_{n}=0\right\} \subset \mathbb{R}^{n}
$$

and let $H_{i j}^{k}$ be the hyperplane $\left\{\overline{\mathbf{x}} \in V \mid x_{i}-x_{j}=k\right\} \subset V$. The hyperplane arrangement $\widetilde{B}_{n}=\left\{H_{i j}^{k}: 0<\right.$ $i<j \leq n, k \in \mathbb{Z}\}$ is called the affine braid arrangement. The connected components of the complement 
to the affine braid arrangement are called alcoves. The group $\widetilde{S}_{n}$ acts on $V$ with the generator $s_{i}$ acting by reflection in the hyperplane $H_{i, i+1}^{0}$ for $i>0$, and $s_{0}$ acting by reflection in the hyperplane $H_{1, n}^{1}$. The action is free and transitive on the set of alcoves, so that the map $\omega \mapsto \omega\left(\mathrm{A}_{0}\right)$, where $\mathrm{A}_{0}:=\left\{\overline{\mathbf{x}} \in V \mid x_{1}>\right.$ $\left.x_{2}>\ldots>x_{n}>x_{1}-1\right\}$ is the fundamental alcove, gives a bijection between the group $\widetilde{S}_{n}$ and the set of alcoves.

Observe $H_{i, j}^{k}=H_{j, i}^{-k}$, so we may always take $i<j$. It is convenient to extend our notation to allow subscripts in $\mathbb{Z}$ via $H_{i+t n, j+t n}^{k}=H_{i, j}^{k}$ and $H_{i, j}^{k}=H_{i, j-n}^{k-1}$. In this way, we can uniquely write each hyperplane in $\widetilde{B}_{n}$ as $H_{i, \ell}^{0}$ with $1 \leq i \leq n, i<\ell, \ell \in \mathbb{Z}$. Then we can define the height of the hyperplane $H_{i, \ell}^{0}$ to be $\ell-i$. Observe, in this manner, the reflecting hyperplane of $s_{0}$ is $H_{1, n}^{1}=H_{1,0}^{0}=H_{0,1}^{0}$ of height 1 . Note that with this notation, the action of the group $\widetilde{S}_{n}$ on the hyperplanes $H_{i, j}^{k}$ is given by $\omega\left(H_{i, j}^{k}\right)=H_{\omega(i), \omega(j)}^{k}$.

There is another way to think about the affine symmetric group:

Definition 2.8 A bijection $\omega: \mathbb{Z} \rightarrow \mathbb{Z}$ is called an affine $S_{n}$-permutation, if $\omega(x+n)=\omega(x)+n$ for all $x$, and $\sum_{i=1}^{n} \omega(i)=\frac{n(n+1)}{2}$.

In this presentation the operation is composition and the generators $s_{1}, \ldots, s_{n-1}, s_{0}$ are given by

$$
s_{i}(x)= \begin{cases}x+1 & \text { for } x \equiv i \bmod n \\ x-1 & \text { for } x \equiv i+1 \bmod n \\ x & \text { otherwise }\end{cases}
$$

It is convenient to use list or window notation for $\omega \in \widetilde{S}_{n}$ as the list $[\omega(1), \omega(2), \cdots, \omega(n)]$. Since $\omega(x+$ $n)=\omega(x)+n$, this determines $\omega$. The bijection between $\widetilde{S}_{n}$ and the set of alcoves can be made more explicit in the following way.

Lemma 2.9 Every alcove A contains exactly one point $\left(x_{1}, \ldots, x_{n}\right)^{T} \in \mathrm{A}$ in its interior such that the numbers $\frac{n+1}{2}-n x_{1}, \ldots, \frac{n+1}{2}-n x_{n}$ are all integers. Moreover, if $\overline{\mathbf{x}} \in \omega\left(\mathrm{A}_{0}\right)$ is such a point, then in the window notation one has

$$
\omega^{-1}=\left[\frac{n+1}{2}-n x_{1}, \ldots, \frac{n+1}{2}-n x_{n}\right] .
$$

These points are called centroids of alcoves.

The minimal length left coset representatives $\omega \in \widetilde{S}_{n} / S_{n}$, also known as affine Grassmannian permutations, satisfy $\omega(1)<\omega(2)<\cdots<\omega(n)$, so that their window notation is an increasing list of integers (summing to $\frac{n(n+1)}{2}$ and with distinct remainders $\bmod n$ ). Their inverses $\omega^{-1}$ are the minimal length right coset representatives and satisfy that the centroids of the alcoves $\omega^{-1}\left(\mathrm{~A}_{0}\right)$ are precisely those whose coordinates are decreasing. That is to say, $\omega^{-1}\left(\mathrm{~A}_{0}\right)$ is in the dominant chamber $\left\{\overline{\mathbf{x}} \in V \mid x_{1}>x_{2}>\cdots>x_{n}\right\}$.

By a slight abuse of notation, we will refer to the set of minimal length left (right) coset representatives as $\widetilde{S}_{n} / S_{n}$ (respectively $S_{n} \backslash \widetilde{S}_{n}$ ).

\subsection{Sommers region}

Definition 2.10 Let $\omega$ be an affine permutation. The set of its inversions is defined as

$$
\operatorname{Inv}(\omega):=\{(i, j) \in \mathbb{Z} \times \mathbb{Z} \mid 1 \leq i \leq n, i<j, \omega(i)>\omega(j)\} .
$$


The length of a permutation $\omega$ is then defined as $\ell(\omega)=\sharp \operatorname{Inv}(\omega)$. This coincides with Coxeter length. We shall say the height of an inversion $(i, j)$ is $j-i$. We will also use the notation $\overline{\operatorname{Inv}}(\omega):=\{(i, j) \in \mathbb{Z} \times \mathbb{Z} \mid$ $i<j, \omega(i)>\omega(j)\}$ for unnormalized inversions.

Geometrically, $\omega$ has an inversion of height $m$ if and only if the alcove $\omega^{-1}\left(\mathrm{~A}_{0}\right)$ is separated from $\mathrm{A}_{0}$ by a (corresponding) hyperplane of height $m$. More precisely, that hyperplane is $H_{i, i+m}^{0}$ if the inversion is $(i, i+m)$. The following definition will play the key role in our constructions:

Definition 2.11 An affine permutation $\omega \in \widetilde{S}_{n}$ is called $m$-stable if for all $x$ the inequality $\omega(x+m)>$ $\omega(x)$ holds, i.e. $\omega$ has no inversions of height $m$. The set of all $m$-stable affine permutations is denoted by $\widetilde{S}_{n}^{m}$.

Definition 2.12 An affine permutation $\omega \in \widetilde{S}_{n}$ is called $m$-restricted if $\omega^{-1} \in \widetilde{S}_{n}^{m}$. We will denote the set of $m$-restricted permutations by ${ }^{m} \widetilde{S}_{n}$. Note $\omega \in{ }^{m} \widetilde{S}_{n}$ if and only if for all $i<j, \omega(i)-\omega(j) \neq m$.

Lemma 2.9 implies an important corollary for the set $\widetilde{S}_{n}^{m}$ :

Lemma 2.13 Let $m=k n+r$, where $0<r<n$. The set of alcoves $\left\{\omega\left(\mathrm{A}_{0}\right): \omega \in m \widetilde{S}_{n}\right\}$ coincides with the set of alcoves that fit inside the region $D_{n}^{m} \subset V$ defined by the inequalities:

1. $x_{i}-x_{i+r} \geq-k$ for $1 \leq i \leq n-r$,

2. $x_{i+r-n}-x_{i} \leq k+1$ for $n-r+1 \leq i \leq n$.

The simplex $D_{n}^{m}$ was first defined in [5, 15] and so we call it the Sommers region. $D_{n}^{m}$ plays the central role in our study. It is worth mentioning that the combinatorial structure of $D_{n}^{m}$ has been recently investigated in [17], where the alcoves in it were labeled by certain sequences of numbers (but not parking functions). We plan to investigate the connections of our work to [17] in the future. It is known that $D_{n}^{m}$ is isometric to the $m$ th dilation of the fundamental alcove. This was proven for all types by Fan [5, Lemma 2.2] and Sommers [15, Theorem 5.7], based on an earlier unpublished observation of Lusztig.

\section{Main Constructions}

\subsection{Bijection $\mathcal{A}: \widetilde{S}_{n}^{m} \rightarrow \mathcal{P} \mathcal{F}_{m / n}$}

We define the map $\mathcal{A}: \widetilde{S}_{n}^{m} \rightarrow \mathcal{P} \mathcal{F}_{m / n}$ by the following procedure. Given $\omega \in \widetilde{S}_{n}^{m}$, consider the set $\Delta_{\omega}:=\{i \in \mathbb{Z}: \omega(i)>0\} \subset \mathbb{Z}$ and let $M_{\omega}$ be its minimal element. Note that the set $\Delta_{\omega}$ is invariant under addition of $m$ and $n$.

Consider the integer lattice $(\mathbb{Z})^{2}$. We prefer to think about it as of the set of square boxes, rather than the set of integer points. Consider the rectangle $R_{m, n}:=\left\{(x, y) \in(\mathbb{Z})^{2} \mid 0 \leq x<m, 0 \leq y<n\right\}$. Let us label the boxes of the lattice according to the linear function

$$
l(x, y):=(m n-m-n)+M_{\omega}-n x-m y
$$

The function $l(x, y)$ is chosen in such a way that a box is labeled by $M_{\omega}$ if and only if its NE corner touches the line containing the NW-SE diagonal of the rectangle $R_{m, n}$, so $l(x, y) \geq M_{\omega}$ if and only if the box $(x, y)$ is below this line. The Young diagram $D_{\omega}$ defined by

$$
D_{\omega}:=\left\{(x, y) \in R_{m, n} \mid l(x, y) \in \Delta_{\omega}\right\}
$$




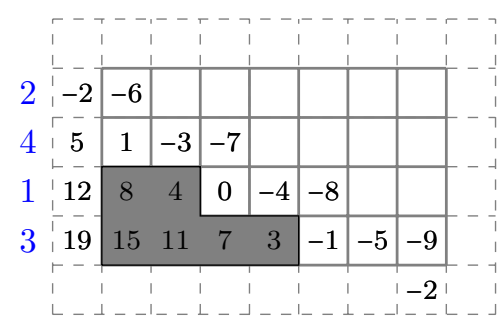

Fig. 2: The labeled diagram corresponding to the permutation $\omega=[0,6,3,1]$.

satisfies $D_{w} \in Y_{m, n}$.

The row-labeling $\tau_{\omega}$ is given by $\tau_{\omega}(i)=\omega\left(a_{i}\right)$, where $a_{i}$ is the label on the rightmost box of the $i$ th row of $D_{\omega}$ (if a row has length 0 we take the label on the box $(-1, i-1)$, just outside the rectangle in the same row). Note that if $i$ th and $(i+1)$ th rows have the same length, then $a_{i+1}=a_{i}-m$ and

$$
\tau_{\omega}(i+1)=\omega\left(a_{i+1}\right)=\omega\left(a_{i}-m\right)<\omega\left(a_{i}\right)=\tau_{\omega}(i) .
$$

Therefore, $\left(D_{\omega}, \tau_{\omega}\right) \in \widehat{Y}_{m, n}$. We define $\mathcal{A}(\omega) \in \mathcal{P F}_{m / n}$ to be the parking function corresponding to $\left(D_{\omega}, \tau_{\omega}\right)$.

Example 3.1 Let $n=4, m=7$. Consider the affine permutation $\omega=[0,6,3,1]=s_{1} s_{0} s_{2} s_{3} s_{2}$ :

$$
\begin{array}{ccccccccccccccc}
x & \ldots & -3 & -2 & -1 & 0 & 1 & 2 & 3 & 4 & 5 & 6 & 7 & 8 & \ldots \\
\omega(x) & \ldots & -4 & 2 & -1 & -3 & 0 & 6 & 3 & 1 & 4 & 10 & 7 & 5 & \ldots
\end{array}
$$

The inversion set is $\operatorname{Inv}(\omega)=\{(2,3),(2,4),(2,5),(2,8),(3,4)\}$. Note that there are no inversions of height 7 , so $\omega$ is 7-stable. Equivalently, $\omega^{-1}=[4,-2,3,5]$ is 7 -restricted. The set $\Delta_{\omega}=\{-2,2,3,4, \ldots\}$ is invariant under the addition of 4 and 7 , and $M_{\omega}=-2$. The diagram $D_{\omega}$ is shown in Figure 2 Note that the labels $3,4,5$, and -2 on the rightmost boxes of the rows of $D_{\omega}$ are the 4-generators of the set $\Delta_{\omega}$, i.e. they are the smallest numbers in $\Delta_{\omega}$ in the corresponding congruence classes $\bmod 4$. It follows then that the corresponding values $\omega(3), \omega(4), \omega(5)$, and $\omega(-2)$ are a permutation of $1,2,3,4$. Indeed, read bottom to top, $(\omega(3), \omega(4), \omega(5), \omega(-2))=(3,1,4,2)$. This defines the row-labeling $\tau_{\omega}:=[3,1,4,2]$. Note that the last (top) two rows of the diagram have the same length 0 . Therefore, the difference between the corresponding labels is $5-(-2)=7$. The 7-stability condition then implies that $\tau(3)=\omega(5)>$ $\omega(-2)=\tau(4)$, which is exactly the required monotonicity condition on the labeling. Using the bijection from Lemma 2.5 one obtains the parking function $\mathcal{A}_{\omega}=(2040)$.

Alternatively, one can define the map $\mathcal{A}$ in a more compact, but less pictorial way:

Definition 3.2 Let $\omega \in \widetilde{S}_{n}^{m}$. We define the corresponding parking function $\mathcal{A}_{\omega}$ as follows. Let $M_{\omega}:=$ $\min \{i \in \mathbb{Z}: \omega(i)>0\}$. Given $\alpha \in\{1, \ldots, n\}$, there is a unique way to express $\omega^{-1}(\alpha)-M_{\omega}$ as a linear combination $r m-k n$ with the condition $r \in\{0, \ldots, n-1\}$. Note that one automatically gets $k \geq 0$. Indeed, otherwise $\alpha=\omega\left(M_{\omega}+r m-k n\right) \geq \omega\left(M_{\omega}\right)-k n>-k n \geq n$, which contradicts the assumption $\alpha \in\{1, \ldots, n\}$. We set $\mathcal{A}_{\omega}(\alpha):=k$.

Lemma 3.3 The two above definitions of the map $\mathcal{A}$ are equivalent. 
Theorem 3.4 The map $\mathcal{A}: \widetilde{S}_{n}^{m} \rightarrow \mathcal{P} \mathcal{F}_{m / n}$ is a bijection.

We call $\mathcal{A}$ the Anderson map, since if we restrict the domain to minimal length right coset representatives (which correspond to partitions called $(m, n)$-cores), and then project to increasing parking functions by sorting, the map agrees with one constructed by Anderson [1].

3.2 Map $\mathcal{P S}: \widetilde{S}_{n}^{m} \rightarrow \mathcal{P} \mathcal{F}_{m / n}$

Definition 3.5 Let $\omega \in \widetilde{S}_{n}^{m}$. Then the map $\mathcal{P} \mathcal{S}_{\omega}:\{1, \ldots, n\} \rightarrow \mathbb{Z}$ is given by:

$$
\mathcal{P} \mathcal{S}_{\omega}(\alpha):=\sharp\left\{\beta \mid \beta>\alpha, 0<\omega^{-1}(\alpha)-\omega^{-1}(\beta)<m\right\}=\sharp\left\{i \mid \omega(i)>\alpha, \omega^{-1}(\alpha)-m<i<\omega^{-1}(\alpha)\right\} .
$$

In other words, $\mathcal{P} \mathcal{S}_{\omega}(\alpha)$ is equal to the number of inversions $(i, j) \in \operatorname{Inv}(\omega)$ of height less than $m$ and such that $\omega(j) \equiv \alpha \bmod n$.

Definition 3.6 Let $\mathcal{S P}:{ }^{m} \widetilde{S}_{n} \rightarrow \mathcal{P} \mathcal{F}_{m / n}$ be defined by $\omega \mapsto \mathcal{P S}_{\omega^{-1}}$. Observe $\mathcal{S P}_{u}(i)=\sharp\{j>i \mid 0<$ $u(i)-u(j)<m\}$.

Example 3.7 Using $\omega=[0,6,3,1]$ as in Example 3.1] one gets $\mathcal{P} \mathcal{S}_{\omega}=(3011)$.

Theorem 3.8 For any m-stable affine permutation $\omega$, the function $\mathcal{P} \mathcal{S}_{\omega}$ is an $m / n$-parking function. Thus one gets a map $\mathcal{P} \mathcal{S}: \widetilde{S}_{n}^{m} \rightarrow \mathcal{P} \mathcal{F}_{m / n}$.

Conjecture 3.9 The map $\mathcal{P S}: \omega \mapsto \mathcal{P} \mathcal{S}_{\omega}$ is a bijection between $\widetilde{S}_{n}^{m}$ and $\mathcal{P} \mathcal{F}_{m / n}$.

In the special cases $m=k n \pm 1$, we can prove that $\mathcal{P} \mathcal{S}$ is a bijection.

It is convenient to extend the domains of the functions $\mathcal{P} \mathcal{S}_{\omega}$ and $\mathcal{S P} \mathcal{P}_{\omega}$ to all integers by using exactly the same formula. Note that in this case $\mathcal{P} \mathcal{S}_{\omega}(\alpha+n)=\mathcal{P} \mathcal{S}_{\omega}(\alpha)$. We have the following results, which should be considered as steps towards Conjecture 3.9 .

Proposition 3.10 Let $\omega \in{ }^{m} \widetilde{S}_{n}$ and let $1 \leq i \leq n, \quad i<j$.

1. $\omega(i)<\omega(i+1) \Longleftrightarrow \mathcal{S P}_{\omega}(i) \leq \mathcal{S P}_{\omega}(i+1)$

2. $(i, j) \in \operatorname{Inv}(\omega) \Longrightarrow \mathcal{S P} \mathcal{P}_{\omega}(i)>\mathcal{S P}_{\omega}(j)$

Proposition 3.11 Let $\omega \in{ }^{m} \widetilde{S}_{n}$.

1. If $0<\omega(i)-\omega(i+1)<m$, then $\left\{\begin{array}{l}\mathcal{S P}_{\omega s_{i}}(i)=\mathcal{S} \mathcal{P}_{\omega}(i+1), \\ \mathcal{S P}_{\omega s_{i}}(i+1)=\mathcal{S} \mathcal{P}_{\omega}(i)-1, \\ \mathcal{S P}_{\omega s_{i}}(j)=\mathcal{S P}_{\omega}(j) \text { for } j \neq \equiv \mid i, i+1 \bmod n\end{array}\right.$

2. If $m<\omega(i)-\omega(i+1)$, then $\left\{\begin{array}{l}\mathcal{S P}_{\omega s_{i}}(i)=\mathcal{S P}_{\omega}(i+1), \\ \mathcal{S P}_{\omega s_{i}}(i+1)=\mathcal{S P}_{\omega}(i), \\ \mathcal{S P}_{\omega s_{i}}(j)=\mathcal{S P}_{\omega}(j) \text { for } j \not \equiv \neq i, i+1 \bmod n\end{array}\right.$

As a corollary to this proposition, we see that $\mathcal{S P}$ is injective on $\left\{\omega \in{ }^{m} \widetilde{S}_{n} \mid(i, j) \in \operatorname{Inv}(\omega) \Longrightarrow\right.$ $\omega(i)-\omega(j)<m\}$. Another interpretation of Proposition 3.10 is that $\mathcal{S P}$ not only respects descents but also respects (weakly) increasing subsequences. 


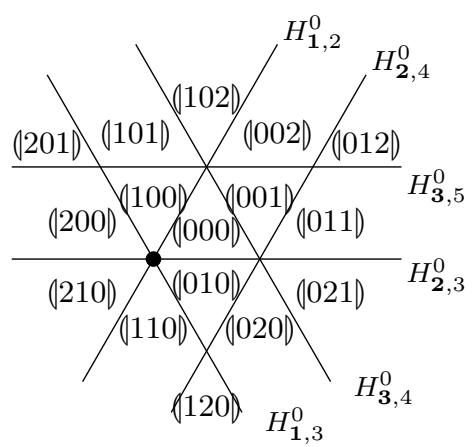

Fig. 3: Pak-Stanley labeling for 1-Shi arrangement for $n=3$.

\section{The cases $m=k n \pm 1$ and the Extended Shi Arrangements}

\subsection{Extended Shi Arrangements and Pak-Stanley Labeling}

Recall the set of $k$-parking functions $\mathcal{P F}_{k}:=\mathcal{P F}_{(k n+1) / n}$. Recall the hyperplanes $H_{i j}^{k}=\left\{\overline{\mathbf{x}} \in V \mid x_{i}-\right.$ $\left.x_{j}=k\right\}$ and the affine braid arrangement $\widetilde{B}_{n}=\left\{H_{i j}^{k} \mid 1 \leq i, j \leq n, k \in \mathbb{Z}\right\}$. The extended Shi arrangement, or $k$-Shi arrangement [14, 16], is defined as a subarrangement of the affine braid arrangement:

Definition 4.1 The hyperplane arrangement

$$
\operatorname{Sh}_{n}^{k}:=\left\{H_{i j}^{\ell}: 1 \leq i<j \leq n,-k<\ell \leq k\right\}=\left\{H_{i j}^{0}: 1 \leq i \leq n, i<j<i+k n, j \neq i \bmod n\right\} .
$$

is called the $k$-Shi arrangement. The connected components of the complement to $\mathrm{Sh}_{n}^{k}$ are called $k$-Shi regions. The set of $k$-Shi regions is denoted $\operatorname{Reg}_{n}^{k}$.

The hyperplane $H_{i j}^{\ell}$ divides $V$ into two half-spaces. Let $H_{i j}^{\ell,<}$ denote the half-space that contains $\mathrm{A}_{0}$ and $H_{i j}^{\ell,>}$ denote the complementary half-space. Note that $H_{i j}^{\ell}$ separates $\omega\left(\mathrm{A}_{0}\right)$ from $\mathrm{A}_{0}$ iff $\omega\left(\mathrm{A}_{0}\right) \subseteq H_{i j}^{\ell,>}$ iff $(i, j-\ell n)$ or $(j, i+\ell n) \in \operatorname{Inv}\left(\omega^{-1}\right)$ (when taking the convention $i, j \in\{1, \ldots, n\}$ ).

Definition 4.2 The Pak-Stanley labeling is the map $\lambda: \operatorname{Reg}_{n}^{k} \rightarrow \mathcal{P} \mathcal{F}_{k}, R \mapsto \lambda_{R}$ defined by the formula

$$
\lambda_{R}(a)=\sharp\left\{H_{i j}^{\ell} \in \mathrm{Sh}_{n}^{k} \mid R \subseteq H_{i j}^{\ell,>}, \ell>0, i=a\right\}+\sharp\left\{H_{i j}^{\ell} \in \mathrm{Sh}_{n}^{k} \mid R \subseteq H_{i j}^{\ell,>}, \ell \leq 0, j=a\right\} .
$$

In other words, one labels the fundamental alcove $\mathrm{A}_{0}$ by the parking function $f=(0 \ldots 0)$, and then as one crosses the hyperplane $H_{i j}^{\ell}$ in the positive direction (i.e. getting further away from $\mathrm{A}_{0}$ ), one adds 1 to $f(j)$ if $\ell \leq 0$ and adds 1 to $f(i)$ if $\ell>0$.

Remark 4.3 One can rewrite this definition as follows:

$$
\lambda_{R}(a)=\sharp\left\{H_{i j}^{0} \in \operatorname{Sh}_{n}^{k} \mid R \subseteq H_{i j}^{0,>}, a=i<j\right\}=\sharp\left\{H_{a a+t}^{0} \mid R \subseteq H_{a a+t}^{0,>}, 0<t<k n, t \equiv \equiv \bmod n\right\} .
$$

We illustrate the Pak-Stanley labeling for $n=3, k=1(m=4)$ in Figure 3

Theorem 4.4 ([16]) The map $\lambda: \operatorname{Reg}_{n}^{k} \rightarrow \mathcal{P F}_{k}$ is a bijection. 

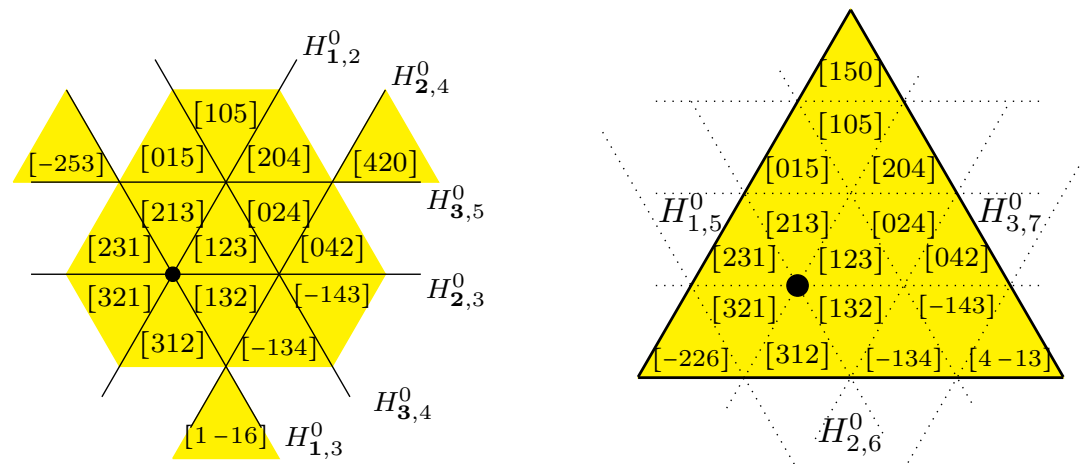

Fig. 4: $m=4, n=3$ On the left, minimal alcoves $\omega\left(\mathrm{A}_{0}\right)$ for $\mathrm{Sh}_{3}^{1}$ are labeled by $\omega \in \widetilde{S}_{n}^{m}$. On the right, alcoves $\omega^{-1}\left(\mathrm{~A}_{0}\right)$ fit inside $D_{3}^{4}$, labeled $\omega^{-1} \in{ }^{m} \widetilde{S}_{n}$.

\subsection{Relation Between Sommers Regions and Extended Shi Arrangements for $m=k n \pm 1$}

Consider the case $m=k n+1$. One can show that each region of an extended Shi arrangement contains a unique minimal alcove (i.e. an alcove with the least number of hyperplanes $H_{i j}^{k}$ separating it from the fundamental alcove $\mathrm{A}_{0}$ ).

Theorem $4.5([\overline{6}])$ An alcove $\omega\left(\mathrm{A}_{0}\right)$ is the minimal alcove of a $k$-Shi region if and only if $\omega^{-1}\left(\mathrm{~A}_{0}\right) \subset$ $D_{n}^{k n+1}$.

See Figure 4. Theorem 4.5 and Lemma 2.13 imply a bijection alc : $\widetilde{S}_{n}^{k n+1} \rightarrow \operatorname{Reg}_{n}^{k}$.

Theorem 4.6 One has $\lambda \circ$ alc $=\mathcal{P} \mathcal{S}$ in this case. In particular, $\mathcal{P S}$ is a bijection for $m=k n+1$.

The case $m=k n-1$ is treated similarly. The main difference is that instead of the set of all $k$-Shi regions $\operatorname{Reg}_{n}^{k}$ one should consider the set of bounded $k$-Shi regions $\widehat{\operatorname{Reg}_{n}^{k}}$. One can show that every bounded $k$-Shi region contains exactly one maximal alcove.

Theorem 4.7 ([7]) An alcove $\omega\left(\mathrm{A}_{0}\right)$ is the maximal alcove of a bounded $k$-Shi region if and only if $\omega^{-1}\left(\mathrm{~A}_{0}\right) \subset D_{n}^{k n-1}$.

As above, we use Lemma 2.13 and Theorem 4.7 to obtain the bijection $\widehat{\text { alc }}: \widetilde{S}_{n}^{k n-1} \rightarrow \widehat{\operatorname{Reg}_{n}^{k}}$. We prove the following theorem:

Theorem 4.8 The image of the subset $\widehat{\operatorname{Reg}_{n}^{k}} \subset \operatorname{Reg}_{n}^{k}$ is exactly $\mathcal{P} \mathcal{F}_{(k n-1) / n} \subset \mathcal{P} \mathcal{F}_{(k n+1) / n}$ under the Pak-Stanley labeling. Furthermore, one gets $\lambda \circ \widehat{\mathrm{alc}}=\mathcal{P} \mathcal{S}$ in this case. In particular, $\mathcal{P} \mathcal{S}$ is a bijection for $m=k n-1$.

\section{Minimal Length Representatives and the Zeta Map}

Definition 5.1 Let $\operatorname{Mod}_{\mathrm{m}, \mathrm{n}}$ be the set of subsets $\Delta \subset \mathbb{Z}_{\geq 0}$, such that $\Delta+m \subset \Delta, \Delta+n \subset \Delta$, and $\min (\Delta)=0$. A number $a$ is called an $n$-generator of $\Delta$, if $a \in \Delta$ and $a-n \notin \Delta$. Every $\Delta \in \operatorname{Mod}_{\mathrm{m}, \mathrm{n}}$ has exactly $n$ distinct $n$-generators. 
In [8, 9] such subsets were called 0-normalized semimodules over the semigroup generated by $m$ and $n$. We will simply call them $m, n$-invariant subsets.

There is a natural map $R: \widetilde{S}_{n}^{m} \rightarrow \operatorname{Mod}_{\mathrm{m}, \mathrm{n}}$ given by $\omega \mapsto \Delta_{\omega}-\min \left(\Delta_{\omega}\right)$ (here, as before, $\Delta_{\omega}=\{i \in \mathbb{Z}$ : $\omega(i)>0\})$. Let $\Omega_{n}^{m}$ be the set of $m$-stable minimal length right coset representatives of $S_{n} \backslash \widetilde{S}_{n}$. In other words,

$$
\Omega_{n}^{m}:=\left\{\omega \in \widetilde{S}_{n}^{m} \mid \omega^{-1}(1)<\ldots<\omega^{-1}(n)\right\} .
$$

One can check that the restriction $\left.R\right|_{\Omega_{n}^{m}}: \Omega_{n}^{m} \rightarrow \operatorname{Mod}_{\mathrm{m}, \mathrm{n}}$ is a bijection. The integers $\omega^{-1}(1), \ldots, \omega^{-1}(n)$ are the $n$-generators of $\Delta_{\omega}$, and since $\omega \in \Omega_{n}^{m}$ we have $\omega^{-1}(1)<\ldots<\omega^{-1}(n)$, so one can uniquely recover $\omega$ from $\Delta_{\omega}$. Let $\hat{R}:=\left.R\right|_{\Omega_{n}^{m}}: \Omega_{n}^{m} \rightarrow \operatorname{Mod}_{\mathrm{m}, \mathrm{n}}$ denote the restriction.

Recall that $Y_{m, n}$ is the set of Young diagrams that fit under diagonal in an $n \times m$ rectangle and $P$ : $\mathcal{P} \mathcal{F}_{m / n} \rightarrow Y_{m, n}$ is the natural map. In [8, 9] the first two named authors constructed two maps $D$ : $\operatorname{Mod}_{\mathrm{m}, \mathrm{n}} \rightarrow Y_{m, n}$ and $G: \operatorname{Mod}_{\mathrm{m}, \mathrm{n}} \rightarrow Y_{m, n}$, proved that $D$ is a bijection, and related the two maps to the theory of $q, t$-Catalan numbers in the following way. In the case $m=n+1$ one gets

$$
c_{n}(q, t)=\sum_{\Delta \in \operatorname{Mod}_{\mathrm{n}+1, \mathrm{n}}} q^{\delta-|D(\Delta)|} t^{\delta-|G(\Delta)|},
$$

where $\delta=\frac{n(n-1)}{2}$ and $c_{n}(q, t)$ is the Garsia-Haiman $q, t$-Catalan polynomial. It is known that these polynomials are symmetric $c_{n}(q, t)=c_{n}(t, q)$, although the proof is highly non-combinatorial and uses the machinery of Hilbert schemes, developed by Haiman. Finding a combinatorial proof of the symmetry of the $q, t$-Catalan polynomials remains an open problem.

The above consideration motivates the rational slope generalization of the $q, t$-Catalan numbers:

$$
c_{m, n}(q, t)=\sum_{\Delta \in \operatorname{Mod}_{\mathrm{m}, \mathrm{n}}} q^{\delta-|D(\Delta)|} t^{\delta-|G(\Delta)|},
$$

where $\delta=\frac{(m-1)(n-1)}{2}$ is the total number of boxes below the diagonal in an $n \times m$ rectangle. The symmetry of these polynomials remains an open problem beyond the classical case $m=n+1$ and the cases $\min (m, n) \leq 4$ (see [9] for $\min (m, n) \leq 3$ and [13] for $\min (m, n)=4$ ). It was also shown in [8] that the composition $G \circ D^{-1}: Y_{m, n} \rightarrow Y_{m, n}$ generalizes Haglund's zeta map exchanging the pairs of statistics (area, dinv) and (bounce, area) on Dyck paths. It was conjectured that the map $G$, and therefore, the generalized Haglund's zeta, are also bijections. This would imply a weaker symmetry property $c_{m, n}(q, 1)=c_{m, n}(1, q)$. In [9] the bijectivity of $G$ was proved for $m=k n \pm 1$. For more details on this work we refer the reader to [8, 9].

Let $\star$ denote the involution on $\widetilde{S}_{n}: \omega^{\star}(x)=1-\omega(1-x)$.

Lemma 5.2 The map $\star$ preserves the set $\widetilde{S}_{n}^{m}$ and the set $\Omega_{n}^{m}$. The map $\nwarrow:(i, j) \mapsto(1-j, 1-i)$ provides a height preserving bijection from $\{(i, j) \mid i<j, \omega(i)>\omega(j)\}$ to $\left\{(i, j) \mid i<j, \omega^{\star}(i)>\omega^{\star}(j)\right\}$.

The following Theorem shows that our constructions are direct generalizations of those of [8, 9]:

Theorem 5.3 One has the following identities:
1. $P \circ \mathcal{A} \circ \hat{R}^{-1}=D$,
2. $P \circ \mathcal{P S} \circ \star \circ \hat{R}^{-1}=G$. 
The involution $\star$ could have been avoided in Theorem 5.3 by adjusting the definition of the map $\mathcal{P S}$. However, in that case one would have to use $\star$ to match the map $\mathcal{P} \mathcal{S}$ for $m=k n+1$ with the Pak-Stanley labeling (see Section 4).

The composition $\mathcal{P} \mathcal{S} \circ \mathcal{A}^{-1}: \mathcal{P} \mathcal{F}_{m / n} \rightarrow \mathcal{P} \mathcal{F}_{m / n}$ should be thought of as a rational slope parking function generalization of the Haglund zeta map $\zeta$. Note that its bijectivity remains conjectural beyond cases $m=k n \pm 1$, which follows immediately from Theorems 4.6 and 4.8

\section{Some examples for $m \neq k n \pm 1$}

In this section we discuss some examples for which $m \neq k n \pm 1$.

Example 6.1 There are $81=3^{4}$ 3/5-parking functions. The $7=\frac{1}{5+3}\left(\begin{array}{c}5+3 \\ 5\end{array}\right)$ increasing parking functions are (00000), (00001), (00002), (00011), (00012), (00111), (00112). Grouping them into the $S_{5}$-orbits $\left\{f \circ \omega \mid \omega \in S_{5}\right\}$ yields $81=1+5+5+10+20+10+30$. There are 7 vectors in $\mathbb{Z}^{5} \cap V \cap D_{5}^{3}$. Their transposes are:

$$
(0,0,0,0,0),(1,0,0,0,-1),(0,1,0,0,-1),(1,0,0,-1,0),(0,0,1,-1,0),(0,1,-1,0,0),(1,-1,0,1,-1)
$$

The 30 parking functions in the $S_{5}$-orbit of $(000112)$ correspond under the map $\mathcal{A}$ to the 30 permutations in $S_{5} \cap{ }^{3} \widetilde{S}_{5}$ which are those in the support of the shuffle $14 \mathrm{w} 25 \mathrm{w} 3$ (that is to say, the intersection of the Sommers region with the orbit of the identity permutation).

On the other hand, the parking function $(00000)=\mathcal{A}\left(\omega_{m}\right)$ corresponds under $\mathcal{A}$ to the affine permutation $\omega_{m}=[-3,0,3,6,9] \in{ }^{3} \widetilde{S}_{5}$. Anything else in its right $S_{5}$-orbit lies outside the Sommers region.

Despite the fact many of the above theorems and constructions use $\widetilde{S}_{n}^{m}$, it is more uniform to study the set $\left\{u \mathrm{~A}_{0} \mid u \in{ }^{m} \widetilde{S}_{n}\right\}$ and $\mathcal{S P}$ than $\left\{\omega \mathrm{A}_{0} \mid \omega \in \widetilde{S}_{n}^{m}\right\}$ and $\mathcal{P} \mathcal{S}$. One reason is that while the Sommers region can always be defined for $\operatorname{gcd}(m, n)=1$, a hyperplane arrangement that is the correct analogue of the Shi arrangement cannot. Consider the following example.

Example 6.2 . In the case $(n, m)=(5,3)$ it is impossible to find a set of hyperplanes that separate the alcoves $\left\{\omega \mathrm{A}_{0} \mid \omega \in \widetilde{S}_{n}^{m}\right\}$ and has $\frac{1}{5+3}\left(\begin{array}{c}5+3 \\ 5\end{array}\right)=7$ dominant regions, i.e. that there are exactly 7 dominant regions with a unique $\omega \mathrm{A}_{0}$ in each. In other words, the notion of $\operatorname{Reg}_{n}^{k}$ does not extend well when $m \neq k n \pm 1$.

Indeed, there are 7 dominant regions corresponding to the 3-restricted affine permutations $\omega \in{ }^{3} \widetilde{S}_{5} \cap$ $\widetilde{S}_{5} / S_{5}$. The hyperplanes $H_{4,6}^{0}, H_{5,6}^{0}$ and $H_{5,7}^{0}$ separate [02346] from other 3-restricted permutations. To separate [-21457] from [-11258], one must add either $H_{3,6}^{0}$ or $H_{5,8}^{0}$ to the arrangement, but this would leave either of the non-3-restricted permutations [-22456] or [01248] in a region with no 3-restricted permutations. Therefore any extension of the classical braid arrangement for $S_{5}$ would either have a region with two 3-restricted permutations or a region with none of them.

\section{References}

[1] J. Anderson. Partitions which are simultaneously $t_{1}$ - and $t_{2}$-core. Discrete Math. 248 (2002), no. $1-3,237-243$. 
[2] D. Armstrong. Hyperplane arrangements and diagonal harmonics. 23rd International Conference on Formal Power Series and Algebraic Combinatorics (FPSAC 2011), 39-50, Discrete Math. Theor. Comput. Sci. Proc., AO, Assoc. Discrete Math. Theor. Comput. Sci., Nancy, 2011.

[3] D. Armstrong, B. Rhoades. The Shi arrangement and the Ish arrangement. Trans. Amer. Math. Soc. 364 (2012), no. 3, 1509-1528.

[4] C. Athanasiadis, S. Linusson. A simple bijection for the regions of the Shi arrangement of hyperplanes. Discrete Math. 204 (1999), no. 1-3, 27-39.

[5] C. K. Fan. Euler characteristic of certain affine flag varieties. Transform. Groups 1 (1996), no. 1-2, 35-39.

[6] S. Fishel, M. Vazirani. A bijection between dominant Shi regions and core partitions. European J. Combin. 31 (2010), no. 8, 2087-2101.

[7] S. Fishel, M. Vazirani. A bijection between (bounded) dominant Shi regions and core partitions, DMTCS Proceedings, 283-294, 2010.

[8] E. Gorsky, M. Mazin. Compactified Jacobians and $q$, $t$-Catalan Numbers, I. Journal of Combinatorial Theory, Series A, 120 (2013) 49-63.

[9] E. Gorsky, M. Mazin. Compactified Jacobians and $q, t$-Catalan Numbers, II. Journal of Algebraic Combinatorics, 39 (2014), no. 1, 153-186.

[10] J. Haglund. The $q, t$-Catalan Numbers and the Space of Diagonal Harmonics: With an Appendix on the Combinatorics of Macdonald Polynomials. AMS University lecture series, 2008.

[11] J. Haglund, M. Haiman, N. Loehr, J. Remmel, A. Ulyanov. A combinatorial formula for the character of the diagonal coinvariants. Duke Math. J., 126 (2005), 195-232.

[12] T. Hikita. Affine Springer fibers of type $A$ and combinatorics of diagonal coinvariants. arXiv: 1203.5878

[13] K. Lee, L. Li, N. Loehr. Combinatorics of certain higher $q, t$-Catalan polynomials: chains, joint symmetry, and the Garsia-Haiman formula. arXiv:1211.2191

[14] J.-Y. Shi. The Kazhdan-Lusztig cells in certain affine Weyl groups. Lecture Notes in Mathematics, no. 1179, Springer-Verlag, Berlin/Heidelberg/New York (1986).

[15] E. Sommers. B-stable ideals in the nilradical of a Borel subalgebra. Canad. Math. Bull., 48(3):460472, 2005.

[16] R. P. Stanley. Hyperplane arrangements, parking functions and tree inversions. Mathematical essays in honor of Gian-Carlo Rota (Cambridge, MA, 1996), 359-375, Progr. Math., 161, Birkhäuser Boston, Boston, MA, 1998.

[17] H. Thomas, N. Williams. Cyclic symmetry of the scaled simplex. J. Algebraic Combin. 39 (2014), no. $2,225-246$. 